\title{
Losing a graph with surface diffusion
}

\author{
Charles M. Elliott and Stanislaus MAIER-PAAPE*
}

(Received January 21, 2000; Revised June 26, 2000)

\begin{abstract}
We construct examples where graphs evolved as curves by a generalized surface diffusion law lose the graph property in finite time, although they remain to exist as smooth curves.
\end{abstract}

Key words: surface diffusion, interface motion, geometric evolution.

\section{Introduction}

We consider geometric evolution laws for curves $\Gamma_{t} \subset \mathbb{R}^{2}, t \geq 0$, which, restricted to $[a, b] \times \mathbb{R}$, where $[a, b]$ is some compact interval in $\mathbb{R}$, are in fact graphs over the $x$-axis. Such kind of situations arise, for example, in surface growth problems $[12,13]$, where the surface of a growing material is modeled by a graph, but the governing evolution equations are geometric.

Our geometric evolution is determined by a generalized surface diffusion law

$$
V=-\varepsilon \partial_{s s} \kappa+f(\kappa, \theta) .
$$

Here $V$ denotes the outward normal velocity of the curve, $\kappa$ is the curvature, $s$ denotes the arclength of the curve and $\theta$ is the angle of the curve with the $x$-axis. $\varepsilon>0$ is a parameter and $f: \mathbb{R}^{2} \rightarrow \mathbb{R}$ is a nonlinear smooth function which is odd in $\kappa$, even and $\pi$-periodic in $\theta$. In the case $f \equiv 0,(1.1)$ is motion by surface diffusion $[3,4,5,10,6]$.

Since the curves $\Gamma_{t}, t \geq 0$, are assumed to be graphs over the $x$-axis, i.e., $\Gamma_{t}=\{(x, h(t, x)), x \in(a, b)\}$, the equation (1.1) becomes

$$
\partial_{t} h=-\varepsilon \partial_{x} \frac{1}{J_{h}} \partial_{x} \kappa_{h}+f\left(\kappa_{h}, \theta_{h}\right), \quad \kappa_{h}=\partial_{x} \frac{1}{J_{h}} \partial_{x} h, \quad x \in[a, b],
$$

where $J_{h}=\left(1+\left(\partial_{x} h\right)^{2}\right)^{1 / 2}$ and $\theta_{h}=\arccos \left(\frac{1}{J_{h}}\right)$ (cf. e.g. [7]). We study

1991 Mathematics Subject Classification : 35K99, 35Q72, 82B24, 82B26.

*Supported by Univ. Augsburg Typ B Forschung 1997, 1998 and by the DFG, Heisenbergstipendium Ma 1587/3-1. 
(1.2) augmented with Neumann and no flux boundary conditions

$$
\partial_{x} h(\cdot, a)=\partial_{x} h(\cdot, b)=\partial_{x} \kappa_{h}(\cdot, a)=\partial_{x} \kappa_{h}(\cdot, b)=0 .
$$

Our main result gives that the evolution laws (1.2) and (1.3) for the graph situation, starting with an initially smooth graph $\Gamma_{0}=\{(x, h(0, x))$, $x \in[a, b]\}$ can lead to a non-graph situation within finite time, although with respect to (1.1) the evolving curves $\Gamma_{t}$ do carry on to exist. In particular, we will show a finite time blow up of $\partial_{x} h$ for some initial graph $\Gamma_{0}$ with a very steep slope at some point.

Our approach also covers the case of periodic boundary conditions, in which case the evolution law (1.2) augmented with the boundary conditions

$$
\partial_{x}^{i} h(\cdot, a)=\partial_{x}^{i} h(\cdot, b), \quad i=0, \ldots, 3,
$$

can also lead to a non-graph situation in finite time.

There are several evolution laws in the literature which use surface diffusion as the leading order term. For instance the law $V=-\varepsilon\left(\partial_{s s} \kappa+\right.$ $\left.\frac{1}{2} \kappa^{3}\right)-\cos (2 \theta) \kappa$ introduced by Davi and Gurtin [4] fits into our setting. Also $V=-\varepsilon \partial_{s s} \kappa-\cos (2 \theta) \kappa$ would be admissible which, in a simplified version $\left(\partial_{s s} \kappa\right.$ substituted by its linearization at $\left.h \equiv 0\right)$, is used by Hunt et al. [11] in a graph situation to model surface growth effects in molecular beam epitaxy. Another equation for surface growth (again in the graph situation) is $V=-\varepsilon \partial_{s s} \kappa-|\cos (\theta)| \kappa$ introduced by Mazor et al. [13]. Here $f$ is not differentiable with respect to $\theta$, but our result would apply for a smooth nearby equation.

The results obtained in this paper are in strong contrast to second order models like the curvature flow equation $V=\kappa$, where the graph-like solutions stay as a graph under periodic boundary conditions. Such a result is easily obtained by the intersection-comparison principle (e.g. [2], [1]).

In Giga [8], among other results it is shown that the graph property is lost in a finite time for $V=\kappa+y$ in the $x y$-plane. There the comparison principle is used. Since the present problem does not have the comparison principle, it is impossible to apply Giga's method here.

Instead, we model the evolution of the curve as the evolution of the distance function with respect to a fixed reference curve which is taken so that it is easy to lose the graph property.

Before we continue with our particular setup, let us briefly remark on 
the conditions imposed on $f$. They are chosen in order to guarantee that certain symmetric curves, evolved by the evolution law (1.1) remain symmetric. We formulate this as a lemma and leave the obvious proof to the reader.

Lemma 1.1 (a) Suppose $f$ is odd in $\kappa$. Then any initially point symmetric curve remains point symmetric under the evolution of (1.1) as long as it exists and is unique.

(b) In case $f$ is even in $\theta$, we obtain that any initially axisymmetric curve remains axisymmetric, as long as it exists and is unique; as axes of symmetry any horizontal or vertical lines are allowed.

We remark that this observation should be sufficient to generalize the pinching of result of Giga and Ito [9] ( $f$ even in $\theta$ is enough) for the generalized surface diffusion law (1.1).

\section{Parameterization and Local Existence}

In the following we use the parameterization introduced by Elliott and Garcke [5] for the evolution in (1.1). The idea is to model the evolution of the curve as the evolution of the distance function with respect to a fixed smooth curve $\mathcal{M}^{*} \subset \mathbb{R}^{2}$, see Figure 1 .

The restriction of $\mathcal{M}^{*}$ to $[-1,1] \times \mathbb{R}$ contains one vertical straight line of length one and two horizontal straight lines of length one half, which are connected smoothly. $\mathcal{M}^{*}$ is then extended to an unbounded smooth curve in $\mathbb{R}^{2}$ by reflection at $\{x=1\}$ and periodic continuation. Let $I=[-L, L] \subset$ $\mathbb{R}$ and let

$$
\begin{aligned}
X^{*}: I & \rightarrow \mathcal{M}^{*} \\
\eta & \mapsto X^{*}(\eta)
\end{aligned}
$$

be an arclength parameterization of $\mathcal{M}^{*} \cap([-2,2] \times \mathbb{R})$ which again may be extended to an arclength parameterization $X^{*}: \mathbb{R} \rightarrow \mathcal{M}^{*}$ of the whole of $\mathcal{M}^{*}$. Then the unit tangent vector $\tau^{*}=\partial_{\eta} X^{*}$ of $\mathcal{M}^{*}$ satisfies the Frenet formulas

$$
\partial_{\eta} \boldsymbol{\tau}^{*}=\kappa^{*} \mathbf{n}^{*}, \quad \partial_{\eta} \mathbf{n}^{*}=-\kappa^{*} \boldsymbol{\tau}^{*},
$$

where $\mathbf{n}^{*}$ is a unit normal vector and $\kappa^{*}$ is the curvature of $\mathcal{M}^{*}$ with the sign convention that curvature of a circle is negative. Another ingredient to 


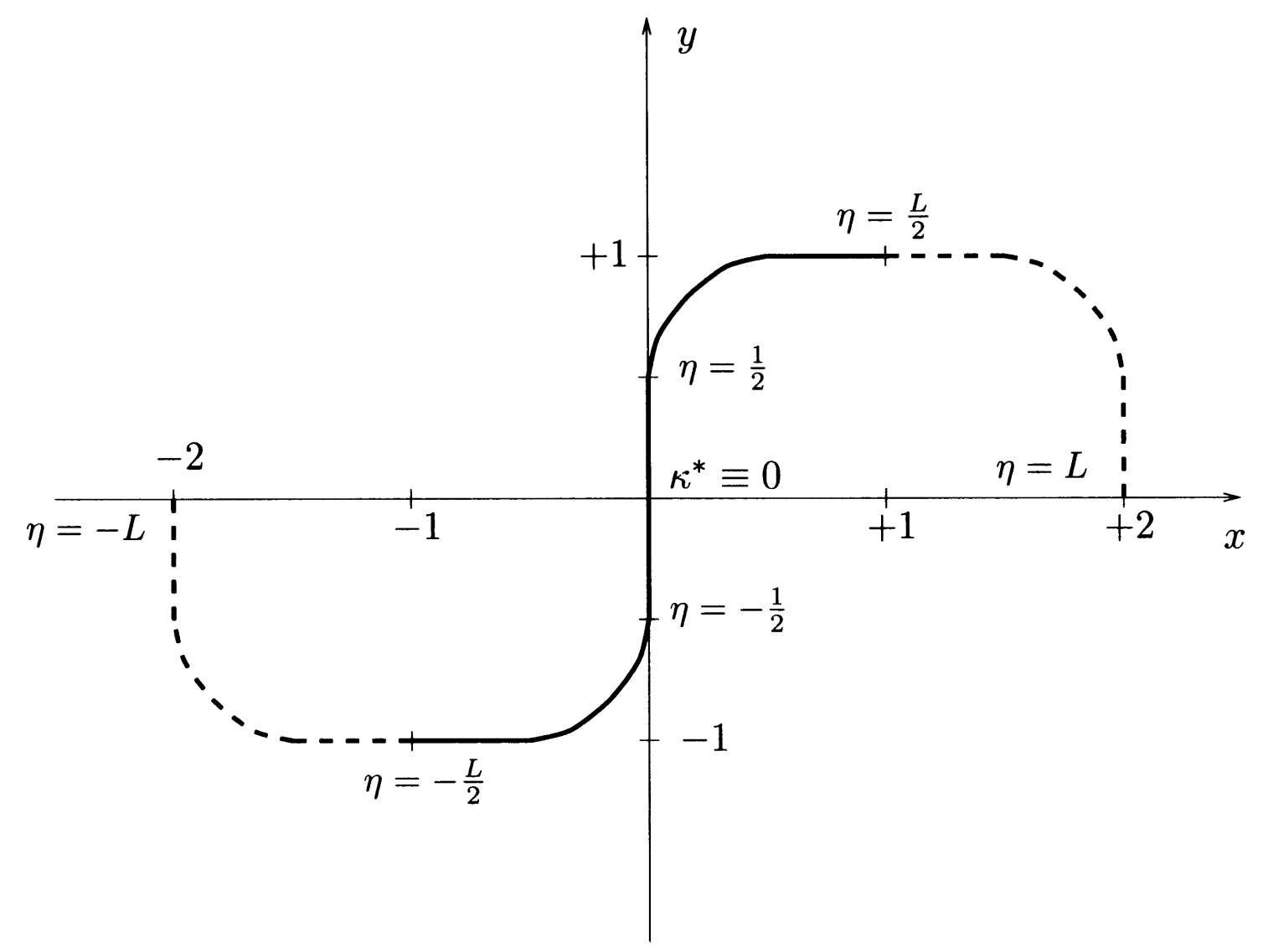

Fig. 1. The reference curve $\mathcal{M}^{*}$.

describe $\mathcal{M}^{*}$ is $\theta^{*}=\theta^{*}(\eta) \in\left[-\frac{\pi}{2}, \frac{\pi}{2}\right]$, the angle of the curve with the $x$-axis.

An initial curve $\Gamma_{0} \subset \mathbb{R}^{2}$, close to $\mathcal{M}^{*}$, subject to (1.1) evolves to $\Gamma_{t} \subset$ $\mathbb{R}^{2}, t \geq 0$. At least for small time $t$ we may parametrize these curves by

$$
\Gamma_{t}(\eta)=X^{*}(\eta)+d(t, \eta) \cdot \mathbf{n}^{*}(\eta), \quad \eta \in \mathbb{R}
$$

where $d$ is the distance function from $\mathcal{M}^{*}$. The tangent $\tau$ and the normal $\mathbf{n}$ of $\Gamma_{t}$ are

$$
\begin{aligned}
& \boldsymbol{\tau}=\frac{1}{J}\left[\left(1-d \kappa^{*}\right) \boldsymbol{\tau}^{*}+d_{\eta} \mathbf{n}^{*}\right] \\
& \mathbf{n}=\frac{1}{J}\left[-d_{\eta} \boldsymbol{\tau}^{*}+\left(1-d \kappa^{*}\right) \mathbf{n}^{*}\right]
\end{aligned}
$$

where $J=\left|\partial_{\eta} \Gamma.\right|=\left(\left(\partial_{\eta} d\right)^{2}+\left(1-d \kappa^{*}\right)^{2}\right)^{1 / 2}$ is the arclength. The evolution law (1.1) now transforms to a differential equation for $d$ 


$$
\frac{1-d \kappa^{*}}{J} \partial_{t} d=-\varepsilon \frac{1}{J} \partial_{\eta}\left(\frac{1}{J} \partial_{\eta} \kappa\right)+f(\kappa, \theta), \quad \eta \in \mathbb{R},
$$

where $\kappa=\kappa(t, \cdot)$ is the curvature of $\Gamma_{t}$ and $\theta=\theta(t, \cdot)$ is the angle of the curve $\Gamma_{t}$ with the $x$-axis: $\cos \theta=\tau_{1}$ with $\tau=\left(\tau_{1}, \tau_{2}\right)$. According to [5] we have

$$
\kappa=\frac{1}{J^{3}}\left\{\left(1-d \kappa^{*}\right) \partial_{\eta}^{2} d+2 \kappa^{*}\left(\partial_{\eta} d\right)^{2}+\partial_{\eta} \kappa^{*} d \partial_{\eta} d+\kappa^{*}\left(1-d \kappa^{*}\right)^{2}\right\}
$$

Therefore, (2.2) is indeed of the form

$$
\partial_{t} d+\varepsilon\left\{J^{-4} \partial_{\eta}^{4} d+P \partial_{\eta}^{3} d+Q\right\}=J \cdot\left(1-\kappa^{*} d\right)^{-1} f(\kappa, \theta), \quad \eta \in \mathbb{R},
$$

where $P$ and $Q$ are with arguments $\left(1-\kappa^{*} d\right)^{-1}, J^{-1}, \partial_{\eta}^{i} \kappa^{*}, i=0, \ldots, 3$, and $\partial_{\eta}^{j} d, j=0,1,2$.

We now come back to the graph situation (1.2). Of course not every curve near $\mathcal{M}^{*}$ is a graph over the $x$-axis, but some are. In fact, being a graph can be viewed as a condition on $d$ (for instance one needs $\partial_{\eta} d<0$ for $\left.\eta \in\left[-\frac{1}{2},+\frac{1}{2}\right]\right)$.

Our goal is to construct an initial smooth curve $\Gamma_{0} \subset \mathbb{R}^{2}$, close to $\mathcal{M}^{*}$, which restricted to $[-1,1] \times \mathbb{R}$ is a graph $\left\{\left(x, h_{0}(x)\right), x \in[-1,1]\right\}$, but when evolved by (1.2) the graph property is lost in finite time. We choose Neumann and no flux boundary conditions

$$
\partial_{x} h(\cdot,-1)=\partial_{x} h(\cdot,+1)=\partial_{x} \kappa_{h}(\cdot,-1)=\partial_{x} \kappa_{h}(\cdot,+1)=0
$$

for this scenario. However, our intention is not to handle this directly in the graph situation, but to monitor the respective evolving curves in the "close to $\mathcal{M}^{*}$ " situation as solutions of (2.4). Therefore we must consider (2.4) for $\eta \in\left[-\frac{L}{2},+\frac{L}{2}\right]$ augmented with the boundary conditions

$$
\partial_{\eta} d\left(\cdot,-\frac{L}{2}\right)=\partial_{\eta} d\left(\cdot,+\frac{L}{2}\right)=\partial_{\eta} \kappa\left(\cdot,-\frac{L}{2}\right)=\partial_{\eta} \kappa\left(\cdot,+\frac{L}{2}\right)=0 .
$$

Next we need a local, i.e., small time, existence result for those curves. In order to be able to use the existence result for the periodic situation due to Giga and Ito [9], we have to extend our problem to periodic boundary conditions. Any smooth curve $\Gamma_{t}$ satisfying (2.6) can be extended by 
reflection at $\{x=-1\}$ and $\{x=+1\}$ to a periodic problem satisfying

$$
\begin{aligned}
\partial_{t} d+\varepsilon\left\{J^{-4} \partial_{\eta}^{4} d+P \partial_{\eta}^{3} d+Q\right\} & =J\left(1-\kappa^{*} d\right)^{-1} f(\kappa, \theta) \quad \text { for } \eta \in I, \\
\partial_{\eta}^{i} d(\cdot,-L) & =\partial_{\eta}^{i} d(\cdot, L) \quad i=0, \ldots, 3 .
\end{aligned}
$$

The local existence theorem below is for solutions $d=d(t, \eta)$ of $(2.7)$ with periodic initial value

$$
d(0, \eta)=d_{0}(\eta), \quad \eta \in I=[-L, L] .
$$

To recover solutions with the boundary condition (2.6) the additional symmetry

$$
\begin{aligned}
& d\left(\cdot,-\frac{L}{2}+\eta\right)=d\left(\cdot,-\frac{L}{2}-\eta\right) \\
& d\left(\cdot,+\frac{L}{2}+\eta\right)=d\left(\cdot,+\frac{L}{2}-\eta\right)
\end{aligned} \quad \text { for } \quad|\eta| \leq \frac{L}{2}
$$

is imposed on the initial value $d_{0}$ and then, due to symmetry of $\mathcal{M}^{*}$ and our equation, conserved for $d$ as long as it exists (cf. Lemma 1.1).

We now rephrase the local existence result of [9]. Since we deal with $2 L$ periodic solutions, we introduce $\mathbf{I}=\mathbb{R} / 2 L \mathbb{Z}$ as our new space for the spatial variable. Let $\mu=\mu(L)$ denote the constant in the Sobolev inequality

$$
\|u\|_{L^{\infty}(\mathbf{I})} \leq \mu\|u\|_{H^{1}(\mathbf{I})} \quad \text { for all } u \in H^{1}(\mathbf{I})
$$

and let $\delta^{*}:=\left(4\left\|\kappa^{*}\right\|_{L^{\infty}(\mathbf{I})}\right)^{-1}>0$. Then [9], Theorem 3 ii) states

Theorem 2.1 ([9]) Let $m \geq 4$ be an integer and $N \in\left(0, \delta^{*} / \mu\right)$. Then there is a uniform existence time $T_{1}(N)>0$, such that for any $d_{0} \in H^{m}(\mathbf{I})$ with $\left\|d_{0}\right\|_{H^{m}(\mathbf{I})} \leq N$, the solution $d=d(t, \eta)$ of (2.7) exists and is unique for $t \in\left[0, T_{1}(N)\right]$. Furthermore,

$$
\begin{aligned}
& d \in L^{2}\left(0, T_{1}(N) ; H^{m+2}(\mathbf{I})\right), \quad \partial_{t} d \in L^{2}\left(0, T_{1}(N) ; H^{m-2}(\mathbf{I})\right) \\
& \text { and }\|d(t, \cdot)\|_{H^{m}(\mathbf{I})} \leq 2 N \quad \text { for } t \in\left[0, T_{1}(N)\right] .
\end{aligned}
$$

\section{Losing the Graph}

We now want to show that an initial graph can develop a vertical slope in finite time, when moved by surface diffusion. To simplify matters we work with odd distance functions $d$

$$
d(\cdot,-\eta)=-d(\cdot, \eta) \text { for } \eta \in \mathbf{I} .
$$


Again oddness of $\mathcal{M}^{*}$ and the symmetry of our equations yield that an initially odd $d_{0}$ will evolve as an odd curve under (2.7) as long as it exists (cf. Lemma 1.1). Oddness immediately implies

$$
\partial_{\eta}^{2 i} d(\cdot, 0)=0 \text { for } i=0,1,2 .
$$

This, together with $\kappa_{\mid\left[-\frac{1}{2}, \frac{1}{2}\right]}^{*} \equiv 0$ enables computations:

$$
\begin{aligned}
\partial_{t \eta} d(t, 0)=\varepsilon & \left\{-\frac{\partial_{\eta}^{5} d(t, 0)}{J(t, 0)^{4}}+10 \frac{\partial_{\eta} d(t, 0) \cdot\left(\partial_{\eta}^{3} d(t, 0)\right)^{2}}{J(t, 0)^{6}}\right\} \\
& +\frac{\partial_{\kappa} f(0, \theta(t, 0)) \partial_{\eta}^{3} d(t, 0)}{J(t, 0)^{2}}=: \sigma(d(t, \cdot)),
\end{aligned}
$$

where $J(t, 0)=\left(1+\left(\partial_{\eta} d(t, 0)\right)^{2}\right)^{1 / 2}$ (cf. (2.2)) $)$. Note that, by the above equation, $\sigma: \mathcal{F} \rightarrow \mathbb{R}$ is well defined, where $\mathcal{F}$ is the set of all sufficiently smooth functions $g: I \rightarrow \mathbb{R}$ (strictly speaking defined on a neighborhood of $0 \in I$ is enough). Both $J$ and $\theta$ are implicitly defined in terms of $g$.

We will find out that a steep graph where $\partial_{t \eta} d_{0}(0)$ is large enough, will steepen and become vertical in finite time. The space of initial values which ensures this situation is

$$
\begin{aligned}
& D_{0}(N, \rho, \tau) \\
& :=\left\{d_{0} \in D(\mathbf{I}) \mid d_{0} \text { corresponds to a graph in } x \in[-1,1]\right. \text {, } \\
& \left.\left\|d_{0}\right\|_{H^{10}(\mathbf{I})} \leq N, \quad \partial_{\eta} d_{0}(0) \geq-\tau \text { and } \sigma\left(d_{0}\right) \geq \rho\right\},
\end{aligned}
$$

where $N, \rho$ and $\tau$ are positive constants and

$$
D(\mathbf{I})=\left\{d \in H^{10}(\mathbf{I}) \mid d \text { is odd and satisfies (2.6) }\right\}
$$

are the functions which obey all the symmetries we have imposed so far. We note that $D_{0}(N, \rho, \tau)$ is nonempty since prototype functions like $d_{0}(\eta)=$ $-c \frac{\eta^{5}}{5 !}-\tau \eta$ for $\eta$ near 0 are contained in $D_{0}(N, \rho, \tau)$ for some $c>0$, at least in case $\rho$ and $\tau$ are small in magnitude compared to $N$.

The local existence Theorem 2.1 guarantees (for small $N$ ) that solutions of (2.7) with initial value $d_{0} \in D_{0}(N, \rho, \tau)$ do exist at least for $t \in$ $\left[0, T_{1}\right], \quad T_{1}=T_{1}(N)$, and $d=d(t, \eta)$ is contained in

$$
\begin{aligned}
& D_{T_{1}}(N)=\left\{d \in L^{2}\left(0, T_{1} ; D(\mathbf{I}) \cap H^{12}(\mathbf{I})\right), \quad \partial_{t} d \in L^{2}\left(0, T_{1} ; H^{8}(\mathbf{I})\right),\right. \\
&\left.\|d(t, \cdot)\|_{H^{10}(\mathbf{I})} \leq 2 N \quad \text { for } t \in\left[0, T_{1}\right]\right\} .
\end{aligned}
$$


For $d \in D_{T_{1}}(N)$ there exists some constant $K=K(N)>0$ such that

$$
\left|\partial_{t t} \partial_{\eta} d(t, 0)\right| \leq K(N) \text { for all } t \in\left[0, T_{1}\right] .
$$

We are now ready to state our main result:

Theorem 3.1 Assume $f: \mathbb{R}^{2} \rightarrow \mathbb{R}$ is smooth, odd in $\kappa$, even and $\pi$ periodic in $\theta$. Then for any $N \in\left(0, \delta^{*} / \mu\right)$ and $\rho, \tau>0$ satisfying

$$
2 K(N) \tau<\rho^{2}<\left(T_{1}(N) K(N)\right)^{2}
$$

there exists some $t_{0}=t_{0}(N, \rho) \in\left(0, T_{1}(N)\right)$, such that the solution $d=$ $d(t, \eta)$ of (2.7) with initial value $d_{0} \in D_{0}(N, \rho, \tau)$ satisfies $\partial_{\eta} d\left(t_{0}, 0\right)>0$.

Proof. Due to our preliminary work, the proof is now easy. We have from (3.2) and the definition of $D_{0}(N, \rho, \tau)$ that

$$
\begin{aligned}
\partial_{\eta} d(t, 0) & =\partial_{\eta} d_{0}(0)+\int_{0}^{t} \partial_{t} \partial_{\eta} d(s, 0) d s \\
& \geq-\tau+t \cdot \sigma\left(d_{0}\right)+\int_{0}^{t} \int_{0}^{s} \partial_{t t} \partial_{\eta} d(u, 0) d u d s \\
& \geq-\tau+\rho \cdot t-\frac{t^{2}}{2} \cdot K(N) \text { for } t \in\left[0, T_{1}(N)\right] .
\end{aligned}
$$

From (3.3) we conclude for $t_{0}:=\frac{\rho}{K(N)} \in\left[0, T_{1}(N)\right]$ that $\partial_{\eta} d\left(t_{0}, 0\right)>0$.

The immediate consequence of Theorem 3.1 is that graphs evolved by surface diffusion may cease to be graphs in finite time. In the particular situation, we have a change from $\partial_{\eta} d(0,0)<0$ to $\partial_{\eta} d\left(t_{0}, 0\right)>0$ giving an infinite slope for the graph since $\mathcal{M}^{*}$ is here vertical. We summarize:

Corollary 3.2 A graph evolved by generalized surface diffusion (1.2) with Neumann and no flux boundary conditions (1.3) can lose the graph property in finite time, while it still evolves as a smooth curve.

Remark 3.3 Since, due to our construction, the considered graphs also satisfy periodic boundary conditions (1.4) in $x \in[-2,2]$, we also obtain examples of periodic graphs that lose the graph property in finite time.

Acknowledgments The second author wishes to thank the University of Sussex for its hospitality during the time this research was carried out. 


\section{References}

[1] Altschuler S., Angenent S. and Giga Y., Mean curvature flow through singularities for surfaces of rotation. J. Geom. Anal. 5 (1995), 293-358.

[2] Angenent S., The zero set of a solution of a parabolic equation. J. Reine Angew. Math. 390 (1988), 79-96.

[3] Cahn J.W. and Taylor J.E., Surface motion by surface diffusion. Acta metall. mater. 42 (1994), 1045-1063.

[4] Davi F. and Gurtin M.E., On the motion of a phase interface by surface diffusion. J. Appl. Math. Phys. (ZAMP), 41 (1990), 782-811.

[5] Elliott C.M. and Garcke H., Existence results for diffusive surface motion laws. Adv. Math. Sci. Appl. 7 (1997), 467-490.

[6] Escher J., Mayer U.F. and Simonett G., The surface diffusion flow for immersed hypersurfaces. SIAM J. Math. Anal. 29 (1998), 1419-1433.

[7] Giaquinta M. and Hildebrandt S., Calculus of Variations I. Vol. 310 of Comp. Stud. Math., Springer, 1996.

[8] Giga Y., Interior derivative blow-up for quasilinear parabolic equations. Discrete Cont. Dyn. Syst. 1 (1995), 449-461.

[9] Giga Y. and Ito K., On pinching of curves moved by surface diffusion. Commun. Appl. Anal. 2(3), (1998), 393-405.

[10] Gurtin M.E., Planar motion of an anisotropic interface. In Motion by mean curvature and related topics (Trento, 1992), de Gruyter, Berlin, 1994, 89-97.

[11] Hunt A.W., Orme C., Williams D.R.M., Orr B.G. and Sander L.M., Instabilities in MBE growth. Europhys. Lett. 27 (1994), 611-616.

[12] Marsili M., Maritan A., Toigo F. and Banavar J.R., Stochastic growth equations and reparametrization invariance. Rev. Mod. Phys. 68 (1996), 963-983.

[13] Mazor A., Srolovitz D.J., Hagan P.S. and Bukiet B.G., Columnar growth in thin films. Phys. Rev. Lett. 60 (1988), 424-427.

Charles M. Elliott

Centre for Mathematical Analysis

and its Applications

School of Mathematical and Physical Sciences

University of Sussex

Falmer, Brighton BN1 9QH, U.K.

E-mail: C.M.Elliott@sussex.ac.uk

Stanislaus Maier-Paape

Institut für Mathematik

Universität Augsburg

Universitätsstraße 14

D-86135 Augsburg, Germany 